\section{Russisches Roulett mit Haselnüssen}

\author{
Eine Nahrungsmittelallergie auf Haselnuss ist in Westeuropa keine \\ Seltenheit, vor allem in Zusammenhang mit einer Birken-Pollinose. \\ Die Betroffenen leben gefährlich, denn Haselnuss ist in Lebens- \\ mittelprodukten ubiquitär.
}

M it doppelblinden, plazebokontrollierten Provokationstests wollten Allergologen der niederländischen Universität Utrecht die Menge an Haselnussprotein ermitteln, auf die Haselnussallergiker reagieren. Dazu untersuchten sie 31 Erwachsene mit positiver Anamnese für Haselnuss-assoziierte allergische Symptome, positivem Pricktest und/oder Haselnuss-spezifischen IgE-Antikörpern > 0,7 kU/l. Die Wissenschaftler servierten standardisierte Mahlzeiten aus gezuckertem Haferbrei, darin enthalten war Haselnussmehl in sieben verschiedenen Dosierungen zwischen $1 \mathrm{mg}$ und $1 \mathrm{~g}$ Haselnussprotein. Dazwischen eingestreut waren randomisiert Plazebo-Portionen ohne Haselnüsse.

Auf die orale Provokation reagierten insgesamt 29 der 31 Probanden.
Erstes Symptom war Jucken im Mund und an den Lippen, in zwei Fällen kam es außerdem zu einem Anschwellen der Lippen, fünfmal traten gastrointestinale Symptome auf, bei einem Probanden kam es zu einer generalisierten Urtikaria. Der Schwellenwert zur Auslösung einer subjektiven Reaktion schwankte zwischen $1 \mathrm{mg}$ und $100 \mathrm{mg}$ Haselnussprotein - entsprechend 6,4-640 mg gemahlene Haselnüsse. Die Extrapolation der Dosis-Reaktions-Kurve ergab, dass $6 \mathrm{mg}$ Haselnussprotein (rund $38 \mathrm{mg}$ Haselnuss) ausreichen, um bei der Hälfte der Haselnuss-allergischen Population Symptome auszulösen.

Fazit: Die Dosis, bei der 50\% der Haselnussallergiker reagieren, entspricht einer Menge, die durchaus in Lebensmittelprodukten versteckt sein kann.

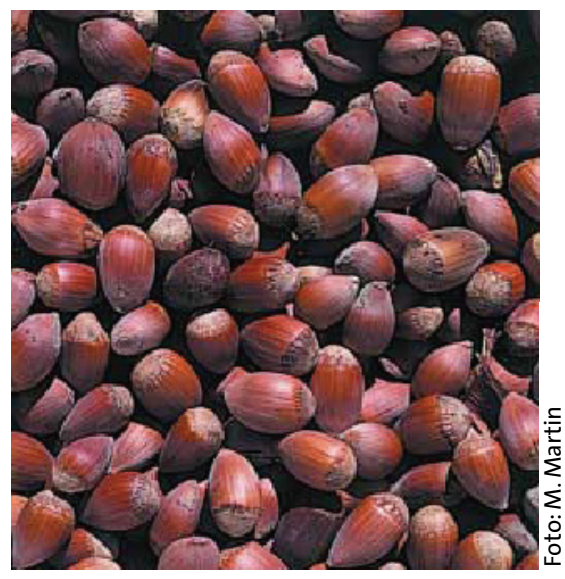

Definitiv mehr als eine kritische Dosis für einen Haselnussallergiker

Solange die Lebensmittelindustrie ihre Produkte nicht gewissenhafter kennzeichnet und Verunreinigungen sicher ausschließt, wird Essen für den Haselnussallergiker ein Vabanquespiel bleiben.

Wensing $\mathbf{M}$ et al. The range of minimum provoking doses in hazelnut-allergic patients as determined by double-blind, placebo-controlled food challenges. Clin Exp Allergy 2002; 32: 1757-62

\title{
Kreuzreaktivität - die Angst isst mit
}

Darf ich Pflaumen essen, wenn ich auf Pfirsiche allergisch bin? Muss Avocado vermieden werden, wenn ich auf Banane reagiere? Bei bestätigten Nahrungsmittelallergien stellt sich für Arzt und Patient das Problem spezifischer Vermeidungsdiäten: zu restriktiv oder zu permissiv?

A llergologen aus Madrid untersuchten 65 Patienten mit IgE-vermittelter Nahrungsmittelallergie immunologisch und klinisch auf mögliche Kreuzreaktivitäten. Patienten mit dokumentierter Allergie gegen Pfirsich, Apfel, Aprikose, Pflaume oder Mandel wurden gegen die verwandten Rosaceae getestet. Bei Reaktion auf Kastanie, Melone, Banane, Kiwi oder Avocado wurde ebenfalls innerhalb dieser Gruppe kreuzgetestet. Probanden mit einer Allergie gegen Erdnüsse, Sonnenblumenkerne, Walnüsse, Pistazien oder Haselnüsse wurden schließlich gegen alle oben genannten Nahrungsmittel getestet.

Insgesamt 223 mögliche immunologische Kreuzreaktivitäten offenbarten sich durch die durchgeführten Pricktests oder durch die Bestimmung von spezifischen IgE-Antikörpern. Nach doppelblinder, plazebokontrollierter oraler Provokation bestätigten sich allerdings nur in 18 Fällen (8\%) bis dahin nicht diagnostizierte klinisch relevante Reaktionen. Diese lagen erwartungsgemäß innerhalb der Gruppen mit verwandten Allergenen. Bei Avocado, Banane, Kiwi, Kastanien und Me- lonen könnten Klasse-I-Chitinasen die gemeinsamen Allergene sein. Klinische Kreuzreaktionen auf Rosaceae könnten auf Homologen von Bet v1 oder von Lipidtransfer-Proteinen beruhen.

Fazit: Kreuzreaktivitäten, die nur bei immunologischen Tests gefunden werden, führen zu einer unnötigen Einschränkung des Speisezettels von Nahrungsmittelallergikern. Die 65 getesteten Patienten hätten demnach unbegründet auf 205 von 223 verschiedenen Früchten, Gemüsen und Nüssen verzichten müssen. Andererseits sollten mögliche Kreuzreaktivitäten durch orale Provokation sicher ausgeschlossen werden, bevor diese Nahrungsmittel ruhigen Gewissens konsumiert werden. c jh

Crespo JF et al. Reactivity to potential cross-reactive foods in fruit-allergic patients: implications for prescribing food avoidance. Allergy 2002; 57: 946-9 